\title{
Single production of vector-like bottom quark at the $\mathrm{LHeC}$
}

\author{
Xue Gong ${ }^{\mathrm{a}}$, Chong-Xing Yue ${ }^{\mathrm{b}}$, Hai-Mei Yuc ${ }^{\mathrm{c}}$, Dong $\mathbf{L i}^{\mathrm{d}}$ \\ Department of Physics, Liaoning Normal University, Dalian 116029, China
}

Received: 2 May 2020 / Accepted: 1 September 2020 / Published online: 22 September 2020

(C) The Author(s) 2020

\begin{abstract}
Existences of vector-like quarks (VLQs) are predicted in many new physics scenarios beyond the Standard Model (SM). We study the possibility of detecting the vector-like bottom quark (VLQ- $B$ ) being the $S U(2)$ singlet with electric charge $-1 / 3$ at Large Hadron electron Collider $(\mathrm{LHeC})$ in a model-independent framework. The decay properties and single production of VLQ- $B$ at the LHeC are explored. Three types of signatures are investigated. By carrying out a fast simulation for the signals and the corresponding backgrounds, the signal significances are obtained. Our numerical results show that detecting of VLQ- $B$ via the semileptonic channel is better than via the fully hadronic or leptonic channel.
\end{abstract}

\section{Introduction}

With the discovery of a $125 \mathrm{GeV}$ Higgs boson in July 2012 by the ATLAS and CMS collaborations at the CERN Large Hadron Collider (LHC) [1,2], the Standard Model (SM) has acquired remarkably success at explaining most of the available experimental phenomena with great accuracy. As yet, there are still unresolved theoretical issues in the SM, such as the nature of the electroweak symmetry breaking and the hierarchy between the electroweak and the Planck scales. One solution is by introducing new heavy particles called vector-like quarks (VLQs) which regulate the Higgs boson mass-squared divergence $[3,4]$. Since VLQs can obtain the gauge invariant mass terms of the form $m \bar{\psi} \psi$ directly, they are not subject to the constraints from Higgs production. Therefore, VLQs as a class of interesting particles have not been excluded by precision measurements.

\footnotetext{
a e-mail: gongxue422@163.com

be-mail: cxyue@1nnu.edu.cn (corresponding author)

ce-mail: yuhaimei233@163.com

de-mail: lidonglnnu@163.com
}

VLQs are hypothetical spin-1/2 colored fermions and are proposed in many new physics scenarios, for example, little Higgs [5-8], composite Higgs [9-12] and extra dimensions models $[13,14]$. The left- and right-handed components of VLQs have the same transformation properties under the SM electroweak symmetry group $[15,16]$. VLQs can be embedded in singlet $[T, B]$, doublets $[(X, T),(T, B)$ or $(B, Y)]$ or triplets $[(X, T, B)$ or $(T, B, Y)]$ for the representations of the $S U(2)$ group. The weak hypercharges of VLQs can be determined by their Yukawa couplings with the SM quarks and the Higgs boson, therefore there are four possible charge assignments: $Q_{T}=+2 / 3, Q_{B}=-1 / 3, Q_{X}=+5 / 3$, and $Q_{Y}=-4 / 3$. In the case of UV complete models, the extra-dimensional models predict a tower of the vector-like top quarks, of which the lightest one has sizable mixing with the third generation quarks [17]. The vector-like bottom quarks are predicted in grand unification theories based on $E_{6}[18,19]$. The doublets $(X, T)$ and $(T, B)$ naturally emerge in warped models [20,21]. The triplets $(X, T, B)$ and $(T, B, Y)$ are predicted in the composite Higgs models [22]. In this paper, we focus on the $S U(2)$ singlet vector-like bottom quark (VLQ- $B$ ) in a model-independent way.

A lot of phenomenological studies for VLQs have been presented in vast literatures [23-38]. Reference [39] has considered single production of VLQ- $B$ which decays into $H b$ at the LHC in context of the composite Higgs model. Reference [40] introduced an effective Lagrangian to study the possibility of detecting VLQ- $B$ via the decay channel $B \rightarrow W t$ at the LHC. Reference [41] performed global fits of the constraints of VLQ- $B$ by using the CKM unitarity violation, excess in Higgs signal strength, and bottom quark forward-backward asymmetry. In our previous work [42], we have considered the capability of detecting VLQ- $B$ at the LHC via single production channel, which is a more potential process than pair production since its less phase-space suppression when the mass of VLQ- $B$ is more heavier.

The concrete search strategies depend on the decay modes of VLQs. If only coupling to the third-generation quarks, the 
vector-like top quark (VLQ- $T$ ) and VLQ- $B$ could decay via the charged current, i.e. $T \rightarrow W b$ and $B \rightarrow W t$, or neutral current, i.e. $T \rightarrow H t, Z t$ and $B \rightarrow H b, Z b$. The subsequent decays of the SM particles produce rich signals. In order to distinguish the type of VLQs, discriminant analysis is required. For example, in the case of fully hadronic final state which is powerful for the $B \rightarrow H b$ channel, reference [43] introduced a discriminant function $P$ that can tag the parent particles of the jets as $W / Z$ boson, Higgs boson or $t$ quark. Together with $b$-tagging and the invariant mass of jets, it is convenient to reduce the background and distinguish signal events of the VLQ- $T$ and VLQ- $B$. Reference [44] combines the searches for pair-produced VLQ- $T$ and VLQ- $B$ at the LHC with all three possible decay modes, the lower limit of singlet VLQ- $B$ mass is a bit larger than that of singlet VLQ- $T$ mass, and the singlet VLQ- $B$ is more sensitivity to the channel $B \rightarrow W t$, while the singlet VLQ- $T$ is more sensitivity to the channel $T \rightarrow H t$.

By now, the direct searches for VLQ- $B$ have been performed by the ATLAS and CMS collaborations at the LHC with center-of-mass (c.m.) energy of $\sqrt{s}=13 \mathrm{TeV}$ and an integrated luminosity of $35-36 \mathrm{fb}^{-1}$ [45-47]. Although there are not any signatures be detected, the constraints on VLQ$B$ have been obtained. The most stringent bounds on the VLQ- $B$ mass are in the range of $700-1800 \mathrm{GeV}$ depending on the production modes, the considered final states and the assumed branching ratios. In fact, the collider has become and will remain an important tool to test wide classes of new physics models. Thus, it is highly motivated to investigate all sensitive search strategies within the possibly available accelerator and detector designs.

Here, we intend to study the possibility of detecting VLQ$B$ in the proposed powerful high energy ep collider, the Large Hadron electron Collider (LHeC) [48] with a $60-140 \mathrm{GeV}$ electron beam and a $7 \mathrm{TeV}$ proton beam from the LHC. It is supposed to run synchronously with the HL-LHC and to deliver the integrated luminosity of $100 \mathrm{fb}^{-1}$ per year and of $1000 \mathrm{fb}^{-1}$ in total. Compared to the previous $e p$ ollider, HERA, the LHeC extends one order of magnitude in the c.m. energy and 1000 times in the integrated luminosity. References [49-51] have studied the discovery potential of the VLQ- $T$ through various channels at the $\mathrm{LHeC}$, where the VLQ- $T$ is the $S U$ (2) singlet with charge $2 / 3$. To the best of our knowledge, so far, no work has been done to search single production of the $S U$ (2) singlet VLQ- $B$ at the LHeC. Hence, we mainly study the observability of the single VLQ$B$ production at the $\mathrm{LHeC}$ combine with the $B \rightarrow W t$ decay channel in our work. Considering the final state has two $W$ bosons (one of those come from top quark decaying), we analyze three types of signatures, which come from the fully hadronic decay channel, the fully leptonic decay channel, and the semileptonic decay channel, respectively. We expect that such work may become a complementary to other production processes in searches for the heavy VLQ- $B$ at the LHeC.

This paper is organized as follows: in Sect. 2, we brief review the couplings of VLQ- $B$ with the SM particles, and discuss its possible decay modes. Section 3 devotes to a detailed analysis of the relevant signals and backgrounds at the LHeC. Finally, we summarize our results in Sect. 4.

\section{Effective Lagrangian and decay modes of the vector-like bottom quark}

VLQs can interact with the SM quarks and the Higgs boson through Yukawa couplings. After the Higgs developing a nonzero vacuum expectation value (VEV), VLQs are allowed to mix with the SM quarks. The mass matrices of quarks are determined by the chosen $S U$ (2) representations of VLQs. By diagonalizing the mass matrices, one can obtain the couplings between physical states which can be found in reference [52]. Reference [53] proposed a more compact parameterization for VLQ- $T$ couplings. Similarly, we consider the same parameterization in the case of VLQ- $B$ and assume that it is the $S U(2)$ singlet. The generic parametrization of an effective Lagrangian of VLQ- $B$ is given by (showing only the couplings relevant for our analysis):

$$
\begin{aligned}
\mathcal{L}= & \frac{\kappa_{B}}{2}\left\{\sqrt{\frac{R_{L}}{1+R_{L}}} \frac{g}{\sqrt{2}}\left[\bar{B}_{L} W_{\mu}^{-} \gamma^{\mu} u_{L}\right]\right. \\
& +\sqrt{\frac{1}{1+R_{L}}} \frac{g}{\sqrt{2}}\left[\bar{B}_{L} W_{\mu}^{-} \gamma^{\mu} t_{L}\right] \\
& +\sqrt{\frac{R_{L}}{1+R_{L}}} \frac{g}{2 C_{W}}\left[\bar{B}_{L} Z_{\mu} \gamma^{\mu} d_{L}\right] \\
& +\sqrt{\frac{1}{1+R_{L}}} \frac{g}{2 C_{W}}\left[\bar{B}_{L} Z_{\mu} \gamma^{\mu} b_{L}\right] \\
& -\sqrt{\frac{R_{L}}{1+R_{L}}} \frac{M_{B}}{v}\left[\bar{B}_{R} H d_{L}\right]-\sqrt{\frac{1}{1+R_{L}}} \frac{M_{B}}{v}\left[\bar{B}_{R} H b_{L}\right] \\
& \left.-\sqrt{\frac{1}{1+R_{L}}} \frac{m_{b}}{v}\left[\bar{B}_{L} H b_{R}\right]\right\}+ \text { h.c., }
\end{aligned}
$$

where $u(d)$ denotes the first or second generation up(down)type quark, $g$ is the $S U$ (2) coupling constant, $v \simeq 246 \mathrm{GeV}$ is the electroweak symmetry breaking scale. We have abbreviated $\cos \theta_{W}$ as $C_{W}$, where $\theta_{W}$ is the Weinberg angle. There are only three parameters that fully describe the relevant interactions we consider. Besides the mass parameter $M_{B}$, there are two coupling parameters appearing in Eq.(1):

- $\kappa_{B}$, the coupling strength to SM quarks in units of standard couplings, which is only relevant to single production; 
- $R_{L}$, the generation mixing coupling, which describes the rate of decays to first two generation quarks with respect to the third generation, where the subscript $L$ represents the chirality of the fermions. For the singlet VLQ- $B$, we neglect the mixing of right-handed quarks since it is suppressed [4].

From Eq. (1) we can see that, for the couplings of the singlet VLQ- $B$ with the SM fermions, $R_{L}=0$ corresponds to coupling to top and bottom quarks only. In many new physics models, VLQs are expected to couple preferentially to thirdgeneration quarks $[15,16]$ and can have flavor-changing neutral-current decays in addition to charged-current decays [44]. While the limit $R_{L}=\infty$ represents coupling to first and second generation of quarks only, which are not excluded $[4,54]$ although not favored.

According to above discussions, VLQ- $B$ has three typical of decay modes: $W u_{i}, Z d_{i}$, and $H d_{i}$, where $i=1,2,3$. The corresponding partial widths are given by

$$
\begin{gathered}
\Gamma\left(B \rightarrow W u_{i}\right)=\frac{c_{i} e^{2} \kappa_{B}^{2} M_{B}^{3}}{256 \pi S_{W}^{2} m_{W}^{2}} \lambda^{1 / 2}\left(1, \frac{m_{u_{i}}^{2}}{M_{B}^{2}}, \frac{m_{W}^{2}}{M_{B}^{2}}\right) \\
\times\left[\left(1-\frac{m_{u_{i}}^{2}}{M_{B}}\right)^{2}+\frac{m_{u_{i}}^{2} m_{W}^{2}}{M_{B}^{4}}+\frac{m_{W}^{2}}{M_{B}^{2}}-2 \frac{m_{W}^{4}}{M_{B}^{4}}\right], \\
\Gamma\left(B \rightarrow Z d_{i}\right)=\frac{c_{i} e^{2} \kappa_{B}^{2} M_{B}^{3}}{512 \pi S_{W}^{2} m_{W}^{2}} \lambda^{1 / 2}\left(1, \frac{m_{d_{i}}^{2}}{M_{B}^{2}}, \frac{m_{Z}^{2}}{M_{B}^{2}}\right) \\
\times\left[\left(1-\frac{m_{d_{i}}^{2}}{M_{B}}\right)^{2}+\frac{m_{d_{i}}^{2} m_{Z}^{2}}{M_{B}^{4}}+\frac{m_{Z}^{2}}{M_{B}^{2}}-2 \frac{m_{Z}^{4}}{M_{B}^{4}}\right], \\
\Gamma\left(B \rightarrow H d_{i}\right)=\frac{c_{i} \kappa_{B}^{2} M_{B}}{128 \pi} \lambda^{1 / 2}\left(1, \frac{m_{d_{i}}^{2}}{M_{B}^{2}}, \frac{m_{h}^{2}}{M_{B}^{2}}\right) \\
\times\left[\left(g_{p}^{2}+g_{m}^{2}\right)\left(1+\frac{m_{d_{i}}^{2}}{M_{B}^{2}}-\frac{m_{h}^{2}}{M_{B}^{2}}\right)+4 g_{m} g_{p} \frac{m_{d_{i}}}{M_{B}}\right],
\end{gathered}
$$

where $c_{i}=2 /\left(1+R_{L}\right)$ for $t, b$ quarks, and $c_{i}=$ $R_{L} /\left(1+R_{L}\right)$ for $u, c, d, s$ quarks,

$g_{m}=\frac{M_{B}}{v}, \quad g_{p}=\frac{m_{b}}{v}$,

and the function $\lambda(a, b, c)$ is given by

$\lambda(a, b, c)=a^{2}+b^{2}+c^{2}-2 a b-2 a c-2 b c$.

In the limit of $M_{B} \gg m_{t}$, the partial widths can be approximate written as

$$
\begin{gathered}
\Gamma\left(B \rightarrow W u_{i}\right) \simeq \frac{c_{i} e^{2} \kappa_{B}^{2} M_{B}^{3}}{256 \pi S_{W}^{2} m_{W}^{2}}, \\
\Gamma\left(B \rightarrow Z d_{i}\right) \simeq \frac{c_{i} e^{2} \kappa_{B}^{2} M_{B}^{3}}{512 \pi S_{W}^{2} m_{W}^{2}},
\end{gathered}
$$

$\Gamma\left(B \rightarrow H d_{i}\right) \simeq \frac{c_{i} \kappa_{B}^{2} M_{B}^{3}}{128 \pi v^{2}}=\frac{c_{i} e^{2} \kappa_{B}^{2} M_{B}^{3}}{512 \pi S_{W}^{2} m_{W}^{2}}$.

From above equations, we can see that, for heavy VLQ$B, \frac{1}{2} \Gamma\left(B \rightarrow W u_{i}\right) \simeq \Gamma\left(B \rightarrow Z d_{i}\right) \simeq \Gamma\left(B \rightarrow H d_{i}\right)$ is a good approximation as expected by the Goldstone boson equivalence theorem [55]. From the Lagrangian given in Eq. (1), one may expect the branching ratios of $H d_{i}$ channel is the largest one since the coupling coefficient of $B H d_{i}$ is proportional to $M_{B}$. Actually, the partial width $\Gamma\left(B \rightarrow H d_{i}\right)$ is proportional to $M_{B}^{3}$, which is similarly with that for other two decay channels as shown in Eqs. (7-9). The branching ratios of these decay channels are plotted as functions of the mass parameter $M_{B}$ and the generation mixing coupling $R_{L}$ in Fig. 1. Since the mass of the boson $W, Z$ or $H$ is much larger than that of the first or second generation quark, the branching ratios of VLQ- $B$ decaying to the first and second generation quarks are approximately equal each other, so we only give the branching ratios of VLQ- $B$ decaying to the first generation quarks in Fig. 1. For $M_{B} \geq 800 \mathrm{GeV}$, one can see that the branching ratios approximate satisfy $\operatorname{Br}\left(B \rightarrow W u_{i}\right)$ : $\operatorname{Br}\left(B \rightarrow Z d_{i}\right): B r\left(B \rightarrow H d_{i}\right) \approx 2: 1: 1$. As expected, the branching ratios of the first and second generation quarks vanish rapidly when $R_{L}$ approaches to zero. When $R_{L}<1$, the third generation quarks plus bosons are the main decay channels. Hence, we choose the $W t$ channel to study the possibility of detecting the signals of VLQ- $B$ at the $\mathrm{LHeC}$ in our work.

\section{Signal analysis and discovery potentiality}

For the single production of VLQ- $B$ at the LHeC, the dominant way is mediated by the exchange of a $W$ or $Z$ boson in the $t$-channel, the $H$-mediated process can be ignored due to the tiny Yukawa coupling between electron and Higgs boson. The relevant Feynman diagrams for the single production and decaying into $W t$ are presented in Fig. 2. For the chosen decay channel of VLQ- $B$, the final state contains two $W$-bosons (one of those coming from top quark decay). There are three types of signatures, which come from the fully hadronic, the fully leptonic and the semileptonic decay channels, respectively.

To proceed further analysis, we need to know the values of some parameters. The SM input parameters which relevant to our calculations are taken from reference [56] as follow:

$m_{t}=173.0 \mathrm{GeV}, \quad m_{Z}=91.1876 \mathrm{GeV}, \quad m_{h}=125 \mathrm{GeV}$,

$S_{W}^{2}=0.231, \quad \alpha\left(m_{Z}\right)=1 / 128$.

Considering the current constraints on the VLQ- $B$ mass [4547], we choose three benchmark points: $M_{B}=800,900,1000$ $\mathrm{GeV}$, which are referred to as $B_{800}, B_{900}, B_{1000}$, respectively. 


$$
-B \rightarrow W t--B \rightarrow W u-B \rightarrow Z b--B \rightarrow Z d-B \rightarrow H b-B \rightarrow H d
$$

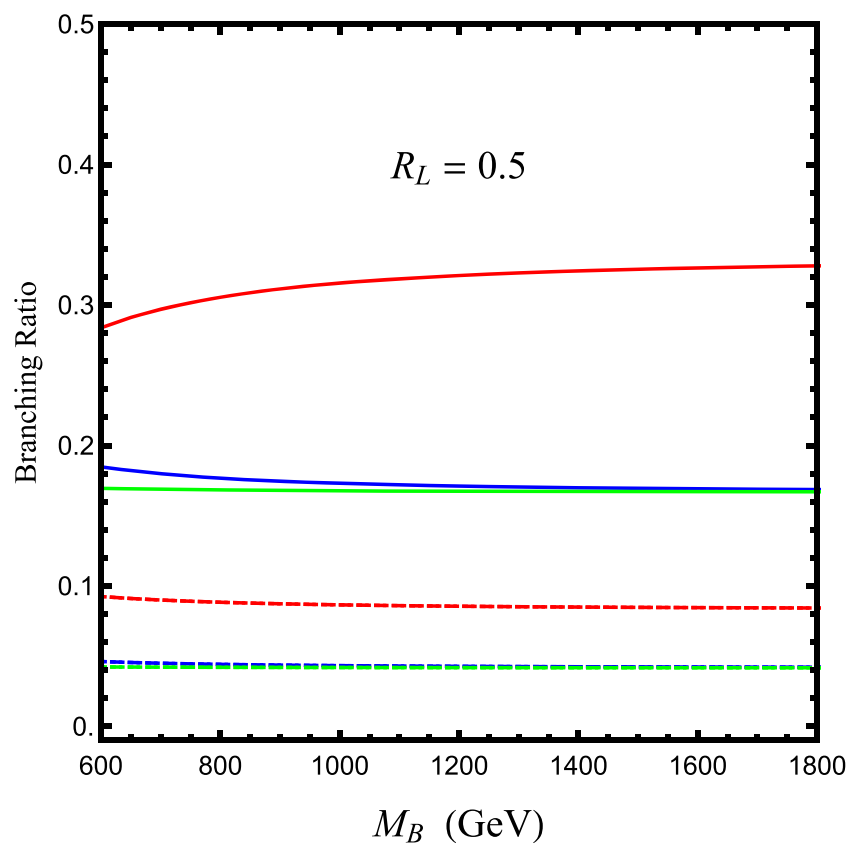

(a)

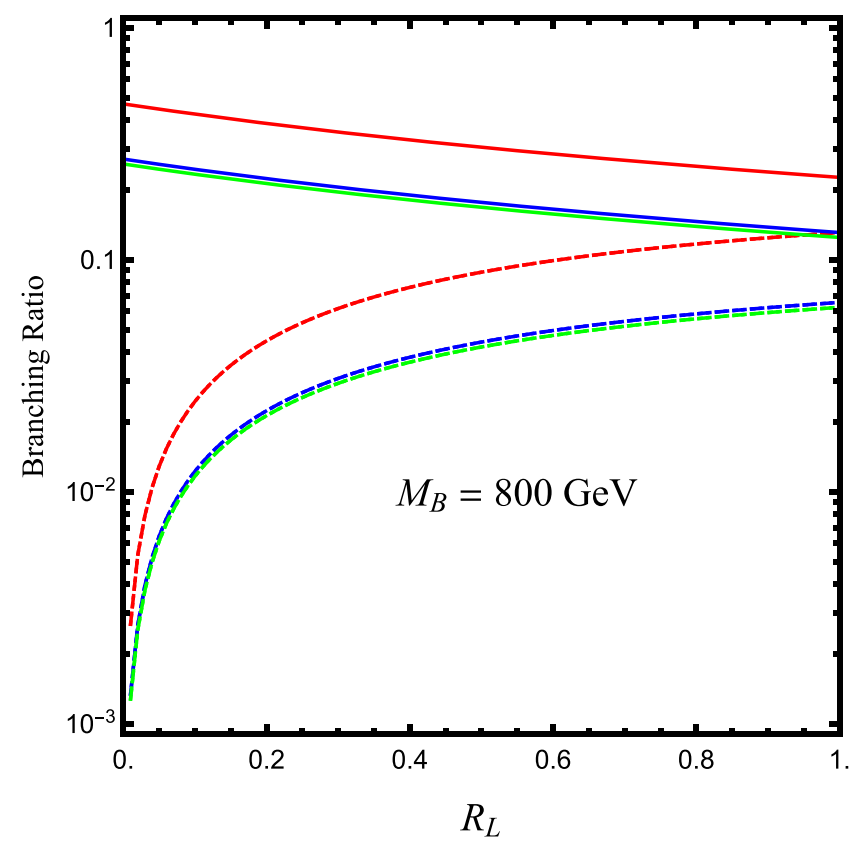

(b)

Fig. 1 The branching ratios of the decay modes $W u_{i}, Z d_{i}$ and $H d_{i}$ as functions of the VLQ- $B$ mass $M_{B}$ (left) and the generation mixing coupling $R_{L}$ (right)
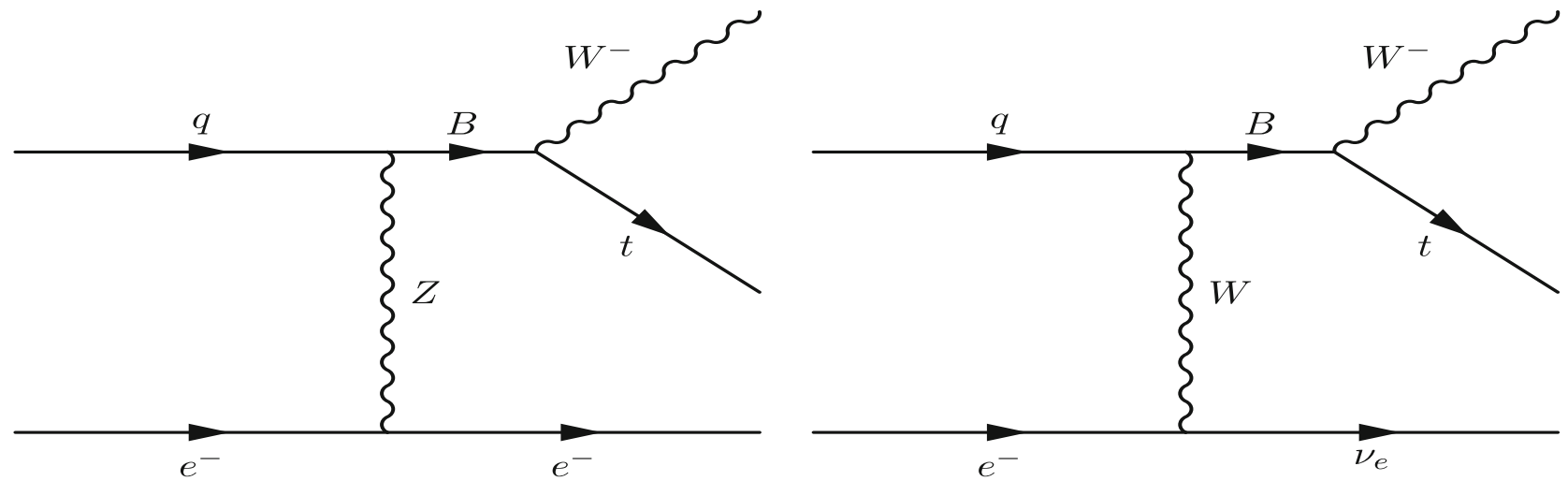

Fig. 2 The Feynman diagrams for single production of VLQ- $B$ at the LHeC including the decay channel $B \rightarrow W t$

The stringent bounds on the coupling parameter $\kappa_{B}$ come from the experimental data about the $Z \bar{b} b$ couplings, which give the upper limit as $\kappa_{B}<0.23$ [53]. For taking the $e^{-}$ and $p$ beam energy as $140 \mathrm{GeV}$ and $7 \mathrm{TeV}$ respectively, the c.m. energy of the LHeC is $\sqrt{s}=1.98 \mathrm{TeV}$. In Figs. 3 and 4 , we show the scaled cross sections of the processes $e^{-} p \rightarrow Z \rightarrow e^{-} B$ and $e^{-} p \rightarrow W \rightarrow v B$ with different $M_{B}, R_{L}$ and $e^{-}$beam polarization at the LHeC. From these figures, one can see that: (1) The cross section of $W$ - mediated process can be enhanced by the polarization of $e^{-}$ beam, while that of $Z$-mediated process is insensitive, since $W$ boson only couple to the left-handed electron. (2) The cross section of $W$-mediated process is more sensitive to $R_{L}$ than that of $Z$-mediated process. And the cross section of $W$-mediated process vanishes when $R_{L}$ approaches to zero, the reason is that no top parton in proton. Hence, in the following, we choose $W$-mediated process for simulation in the case of $P_{e}=-0.8, \kappa_{B}=0.1$, and $R_{L}=0.5$. 


$$
\begin{array}{ll}
--R_{L}=0.01, P_{e}=0 & --R_{L}=0.5, P_{e}=0 \\
-R_{L}=0.01, & P_{e}=-0.8
\end{array}
$$

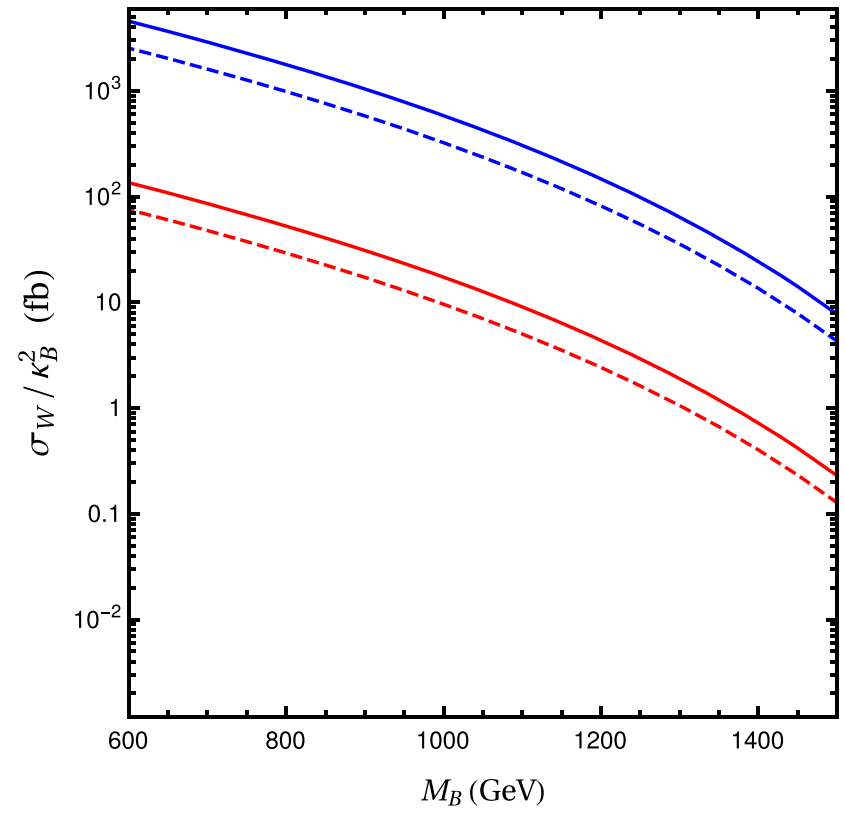

(a)

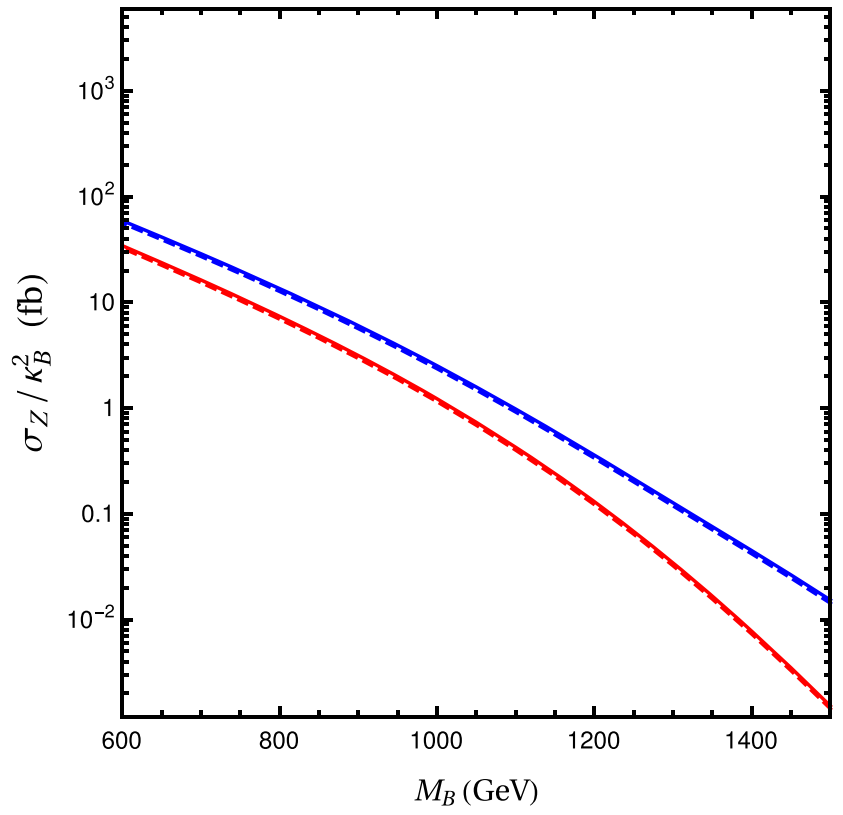

(b)

Fig. 3 The scaled cross sections of the processes $e^{-} p \rightarrow W \rightarrow v B$ and $e^{-} p \rightarrow Z \rightarrow e^{-} B$ as functions of $M_{B}$ for different $R_{L}$ and polarization of $e^{-}$beam

The model file [57] which realize the Lagrangian given by Eq. (1) can be found in the dedicated FeynRules package [58-60]. Signal and background events are simulated at the leading order using MadGraph5-aMC@NLO [61,62] with the CTEQ6L parton distribution function (PDF) [63]. Showering, fragmentation and hadronization are performed with customized Pythia [64]. The PGS is applied for detector simulation, and the relevant parameters are taken for $\mathrm{LHeC}$ Detector Design [48,65]. The anti- $\kappa_{t}$ algorithm [66] with parameter $\Delta R=0.4$ is used to reconstruct the jets. Finally, MadAnalysis5 [67-69] is applied for data analysis and plotting. The energy resolutions of lepton and jets are taken as [48]

$\frac{\Delta E}{E}=\frac{a}{\sqrt{E}} \oplus b$,

where $a=0.45 \mathrm{GeV}^{-1}, b=0.03$ for jets and $a=$ $0.085 \mathrm{GeV}^{-1}, b=0.003$ for leptons. Assuming the efficiencies of $\mathrm{LHeC}$ detector are the same with those of CMS detector, then the efficiency of muon is set as 0.95 , the efficiency of electron is set as 0.95 for $|\eta| \leq 1.5$ and 0.85 for $1.5 \leq|\eta| \leq 2.5$. The efficiency for tagging $b$-quark jets is $85 \%$, the light-parton misidentification probability is $10 \%$ [70].

\subsection{The fully hadronic channel}

In this subsection, we analyze the signal and background events and explore the sensitivity of the singlet VLQ- $B$ at the $\mathrm{LHeC}(\sqrt{s}=1.98 \mathrm{TeV})$ through the fully hadronic decay channel:

$$
\begin{aligned}
e^{-} p & \rightarrow v B\left(\rightarrow W^{-} t\right) \rightarrow v W^{-}(\rightarrow j j) t\left(\rightarrow W^{+} b \rightarrow j j b\right) \\
& \rightarrow 4 j+b+E_{T} .
\end{aligned}
$$

For this channel, the typical signal is one $b$-jet, four jets and large missing energy. The main SM backgrounds come from the following five processes:

- BKG1: $v \bar{t}+$ jets: $v p \rightarrow v \bar{t}+$ jets $\rightarrow v W^{-} \bar{b}+$ jets $\rightarrow$ $\bar{b}+E_{T}+$ jets,

- BKG2: $\bar{v} V+$ jets: $e^{-} p \rightarrow v V+$ jets $\rightarrow E_{T}^{\prime}+$ jets,

- BKG3: $v t \bar{t}+$ jets: $v p \rightarrow v t \bar{t}+$ jets $\rightarrow v W^{-} \bar{b} W^{+} b+$ jets $\rightarrow b \bar{b}+E_{T}+$ jets,

- BKG4: $\bar{v} V V+$ jets: $e^{-} p \rightarrow v V V+$ jets $\rightarrow E_{T}+$ jets,

- BKG5: $\bar{v} V \bar{t}+$ jets: $e^{-} p \rightarrow v V \bar{t}+$ jets $\rightarrow v V W^{-} \bar{b}+$ jets $\rightarrow \bar{b}+E_{T}+$ jets, 


$$
\begin{array}{ll}
--M_{B}=800 \mathrm{GeV}, P_{e}=0 & --M_{B}=1000 \mathrm{GeV}, P_{e}=0 \\
-M_{B}=800 \mathrm{GeV}, P_{e}=-0.8 & -M_{B}=1000 \mathrm{GeV}, P_{e}=-0.8
\end{array}
$$

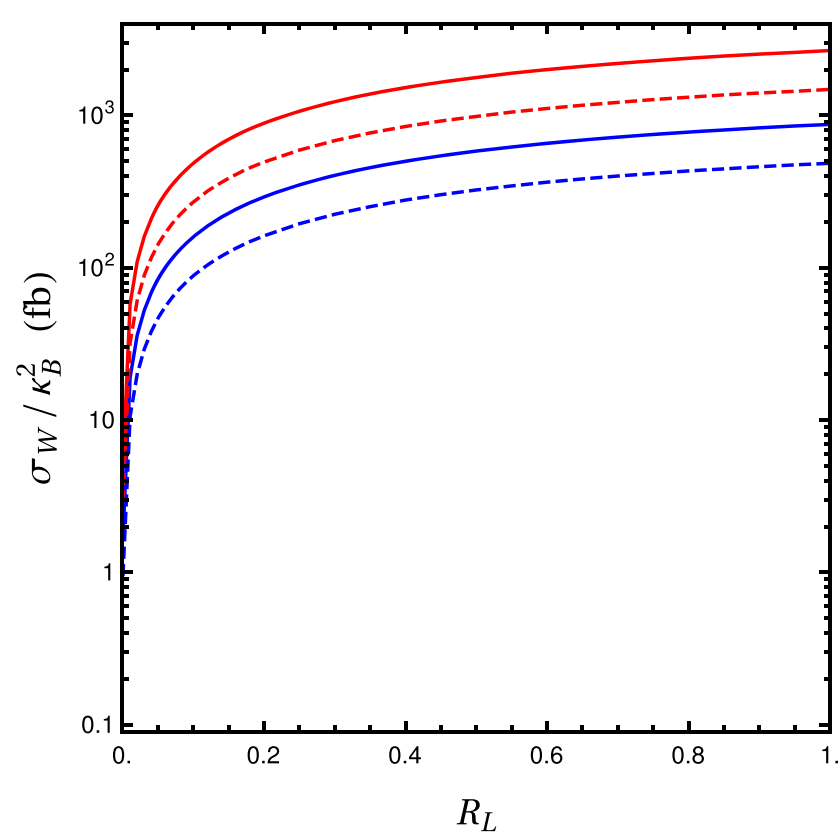

(a)

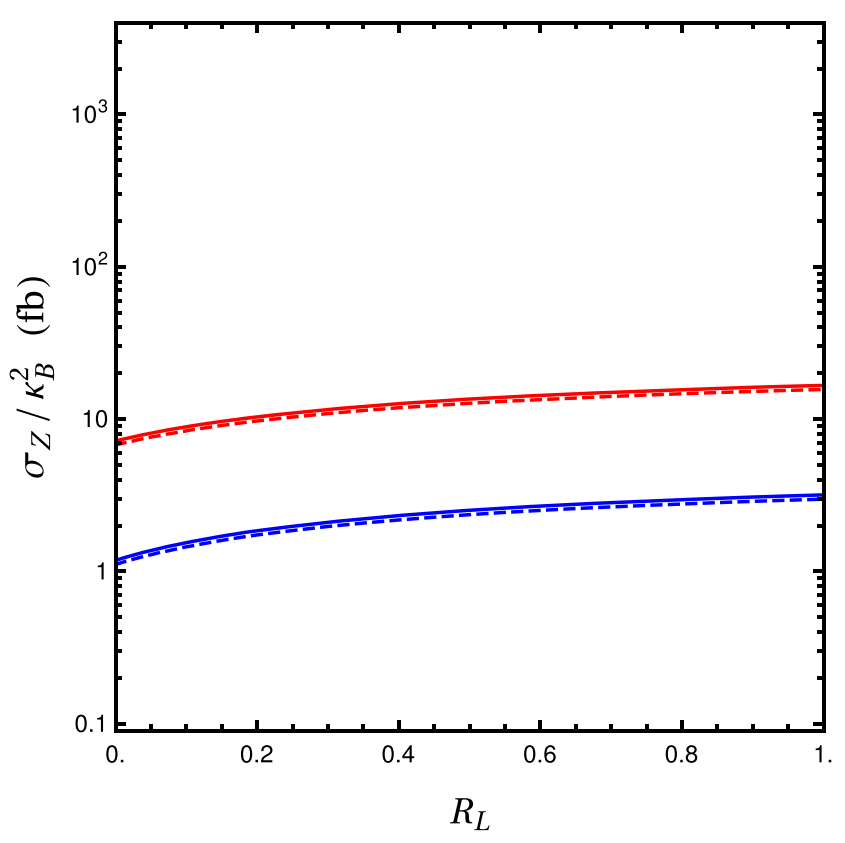

(b)

Fig. 4 The scaled cross sections of the processes $e^{-} p \rightarrow W \rightarrow v B$ and $e^{-} p \rightarrow Z \rightarrow e^{-} B$ as functions of $R_{L}$ for different $M_{B}$ and polarization of $e^{-}$beam

where $V$ denotes $W$ or $Z$ boson, the one light jet mentioned above can be faked as $b$-jet. To avoid double counting for multiple jets and parton shower, the MLM [71] matching method with xqcut $=25 \mathrm{GeV}$ is applied. The signal and background processes are simulated at the $\mathrm{LHeC}$ with the integrated luminosity of $1000 \mathrm{fb}^{-1}$. Firstly, we apply the basic cuts to the signal and background events, which are used to simulate the geometrical acceptance and detection threshold of the detector. These basic cuts are selected as follows in our simulation

$p_{T}^{j}>20 \mathrm{GeV}, \quad\left|\eta^{j}\right|<5, \quad \Delta R(x, y)>0.4$,

where the particle separation $\Delta R_{x y}$ is defined as $\sqrt{\left(\Delta \eta_{x y}\right)^{2}+\left(\Delta \phi_{x y}\right)^{2}}$ with $\Delta \eta_{x y}$ and $\Delta \phi_{x y}$ being the rapidity and azimuthal angle gaps between the two particles $x$ and $y$.

We use the characteristics of the signal as a handle to reduce the backgrounds. Hence, we dipict the normalized distributions of $p_{T}^{j}, \Delta R_{j_{1}, j_{2}}, M_{j_{1}, j_{2}}$ and $H_{T}$ for signals and backgrounds in Fig. 5. According to the information of these kinematic distributions, we impose the following cuts to get a high statistical significance. All cuts applied are given in the following list.
- Cut 1: The first kinematical selection involves the transverse momentum of jet $p_{T}^{j}$, which is shown in Fig. 5a for signals and backgrounds. Since the jets of signal come from the boosted $W$ bosons, they have a larger transverse momentum. Thus, events with $p_{T}^{j}>150 \mathrm{GeV}$ are selected.

- Cut 2: The distributions of the transverse momentum $\Delta R_{j_{1}, j_{2}}$ of the jets for signals and backgrounds are shown in Fig. 5b. Based on these normalized distributions, we require the second cut selection is $R_{j_{1}, j_{2}}>2.5$.

- Cut 3: Figure 5c, d show the normalized distributions of $M_{j_{1}, j_{2}}$ and $H_{T}$, where $M_{j_{1}, j_{2}}$ is the invariant mass of two jets and $H_{T}$ denotes the scalar sum of the transverse momenta of the $b$-tagged jet, the untagged jet and the charged electron. Since the jets of signals all come from the massive VLQ- $B$, the $M_{j_{1}, j_{2}}$ and $H_{T}$ peaks of signal and background are separate. Then the cuts $M_{j_{1}, j_{2}}>$ $350 \mathrm{GeV}$ and $H_{T}>450 \mathrm{GeV}$ are imposed.

In order to see whether the signatures of VLQ- $B$ can be detected at the $\mathrm{LHeC}$, we further calculate the statistical significance of signal events:

$S S=\frac{S}{\sqrt{S+B}}$, 


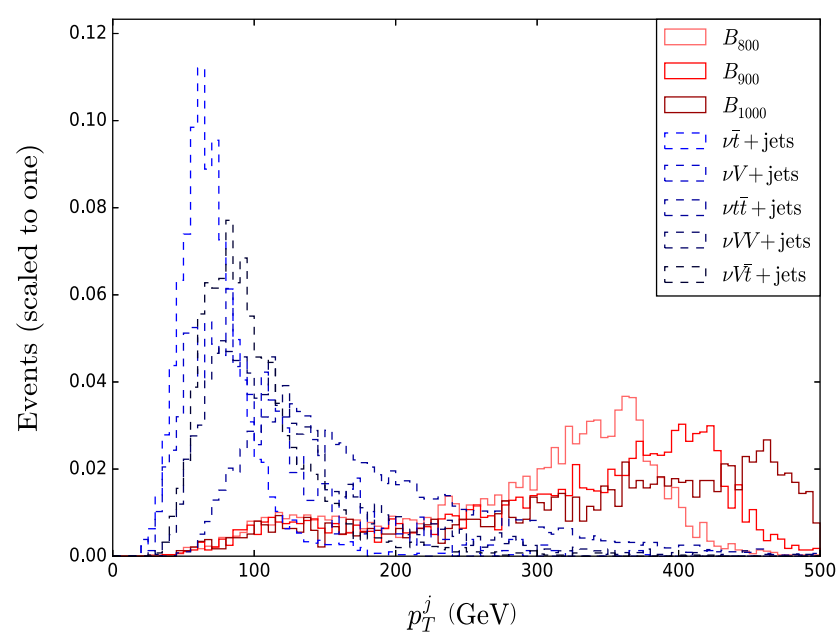

(a)

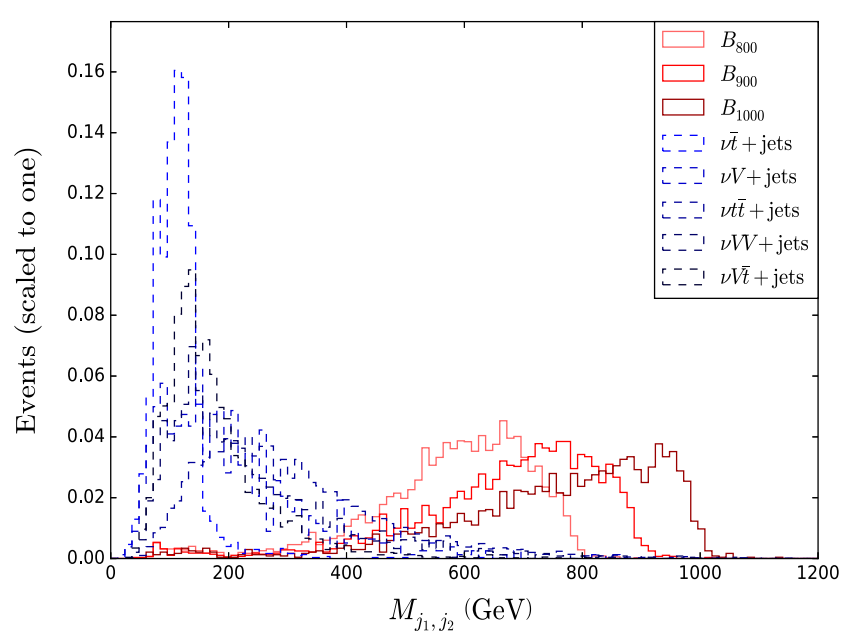

(c)

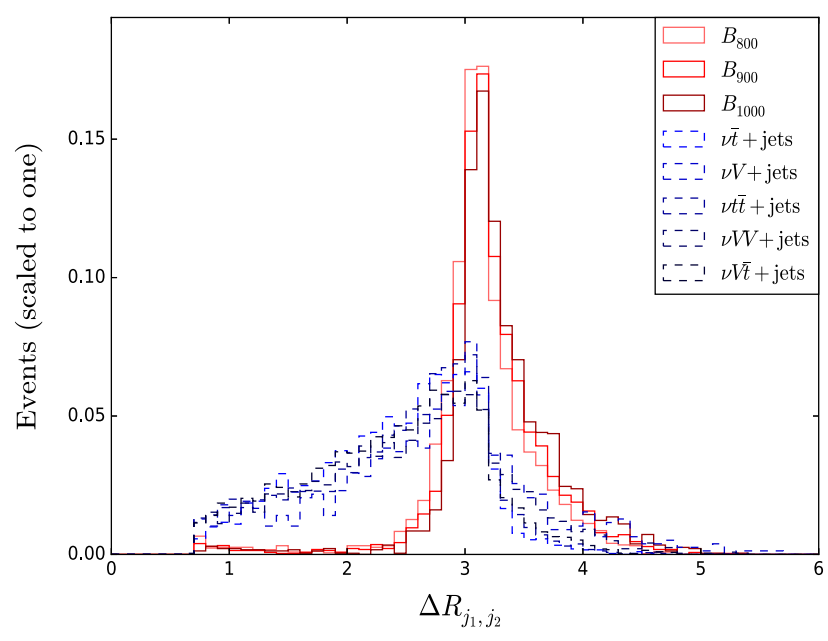

(b)

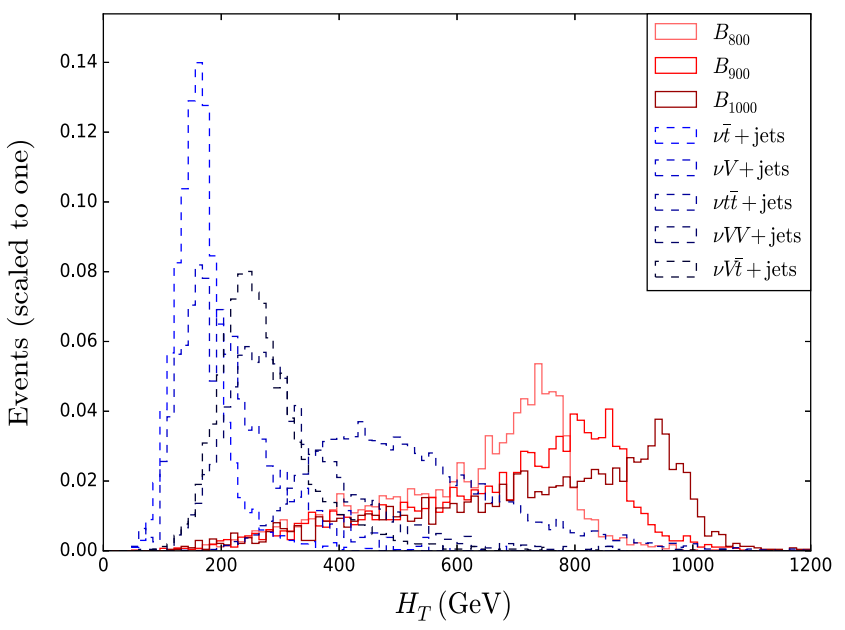

(d)

Fig. 5 Normalized distributions of $p_{T}^{j}, \Delta R_{j_{1}, j_{2}}, M_{j_{1}, j_{2}}$ and $H_{T}$ for signals and backgrounds at the LHeC $(\sqrt{s}=1.98 \mathrm{TeV})$ with the integrated luminosity of $1000 \mathrm{fb}^{-1}$

Table 1 Numbers of the signal and background events at the $\operatorname{LHeC}(\sqrt{s}=1.98 \mathrm{TeV})$ with the integrated luminosity $\mathcal{L}=1000 \mathrm{fb}^{-1}$. Here, we take the coupling parameter $\kappa_{B}=0.1$ and $R_{L}=0.5$

\begin{tabular}{|c|c|c|c|c|c|c|c|c|c|}
\hline & \multicolumn{3}{|c|}{$\underline{\text { Signals }}$} & \multicolumn{6}{|c|}{$\underline{\text { Backgrounds }}$} \\
\hline & $B_{800}$ & $B_{900}$ & $B_{1000}$ & $v \bar{t}+$ jets & $v V+$ jets & $v t \bar{t}+$ jets & $v V V+$ jets & $v V \bar{t}+$ jets & Total \\
\hline Basic cuts & 242.1 & 110.0 & 53.3 & 546992 & 66100 & 31.8 & 2018.5 & 1001.6 & 616144 \\
\hline Cut 1 & 211.6 & 99.4 & 48.5 & 11607 & 11679 & 17.7 & 501.7 & 102.5 & 23908 \\
\hline Cut 2 & 205.7 & 97.6 & 47.6 & 7073 & 8125 & 10.8 & 343.6 & 62.9 & 15615 \\
\hline Cut 3 & 203.9 & 97.3 & 47.4 & 725.5 & 1015.6 & 7.4 & 144.8 & 18.6 & 1911.8 \\
\hline
\end{tabular}

where $S$ and $B$ denote the numbers of the signal and background events, respectively. We define $S S=5$ and 3 as the discovery significance and the possible evidence, respectively. In Table 1, we show the numbers of the signal and background events at the $\operatorname{LHeC}(\sqrt{s}=1.98 \mathrm{TeV})$ with the integrated luminosity $\mathcal{L}=1000 \mathrm{fb}^{-1}$. From the numerical results, we can see that the relevant backgrounds are suppressed effectively, while the signals still have a relatively good efficiency after imposing the above selection cuts. The 


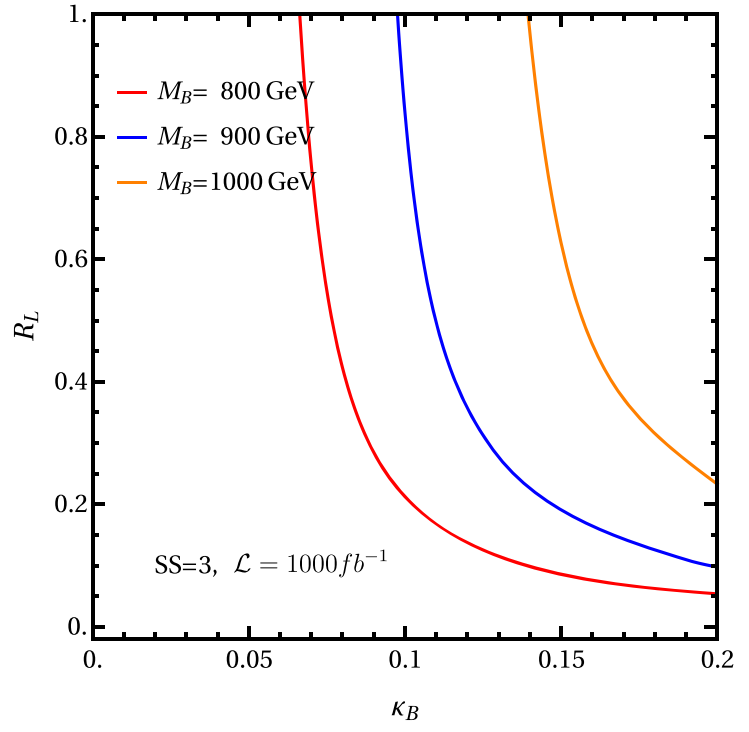

(a)

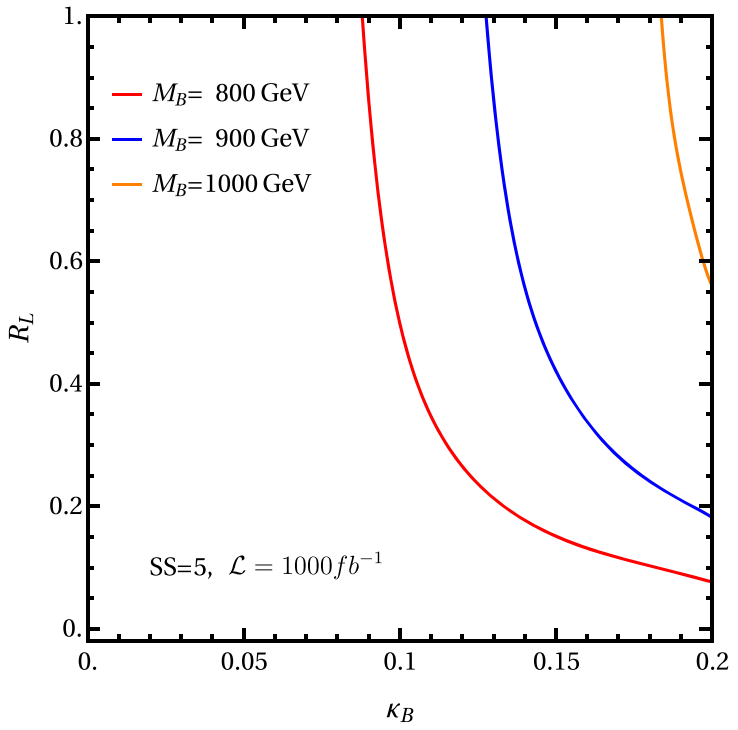

(b)

Fig. $63 \sigma$ (left) and $5 \sigma$ (right) contour plots in the $R_{L}-\kappa_{B}$ plane with three typical VLQ-B masses at the LHeC with the integrated luminosity of $1000 \mathrm{fb}^{-1}$

values of SS can respectively reach about 4.4, 2.2 and 1.0 at the $\mathcal{L}=1000 \mathrm{fb}^{-1}$ for $M_{B} x=800,900$ and $1000 \mathrm{GeV}$.

For the purpose of investigating the signal of the singlet VLQ- $B$ more comprehensively, we show the $3 \sigma$ (left) and $5 \sigma$ (right) contour plots in the $R_{L}-\kappa_{B}$ plane with three typical VLQ- $B$ masses at the LHeC with the integrated luminosity $\mathcal{L}=1000 \mathrm{fb}^{-1}$ in Fig. 6. One can see that, the statistical significance can reach $3 \sigma$ when the values of $\kappa_{B}$ is about $0.076,0.110$ and 0.156 for $R_{L}=0.5$ and $M_{B}=800,900$ and $1000 \mathrm{GeV}$ respectively.

\subsection{The fully leptonic channel}

Similar with the previous section, we analyze the observation potential and explore the sensitivity of the singlet VLQ- $B$ at the $\mathrm{LHeC}$ through the leptonic decay channel:

$$
\begin{aligned}
e^{-} p & \rightarrow v B\left(\rightarrow W^{-} t\right) \rightarrow v W^{-}\left(\rightarrow l_{i}^{-} \bar{v}_{i}\right) t\left(\rightarrow W^{+} b \rightarrow l_{j}^{+} v_{j} b\right) \\
& \rightarrow l_{i}^{-} l_{j}^{+}+b+\mathbb{E}_{T} .
\end{aligned}
$$

For this channel, the typical signal is exactly two charged leptons, one $b$ jet, and missing energy. The main SM backgrounds come from the following processes:

- BKG1: $v \bar{t}+$ jets: $e^{-} p \rightarrow v \bar{t}+$ jets $\rightarrow v W^{-} \bar{b}+$ jets $\rightarrow$ $l^{-}+\bar{b}+\mathbb{E}_{T}+$ jets,

- BKG2: $v V+$ jets: $e^{-} p \rightarrow v V+$ jets $\rightarrow\left(l^{-}\right.$or $\left.l^{-} l^{+}\right)+$ $\mathbb{E}_{T}+$ jets,

- BKG3: $v V V+$ jets: $e^{-} p \rightarrow v V V+$ jets $\rightarrow(2 l, 3 l$ or $4 l)+$ $\mathbb{E}_{T}+$ jets,
- BKG4: $v V \bar{t}+$ jets: $e^{-} p \rightarrow v Z \bar{t}+$ jets $\rightarrow v l^{-} l^{+} W^{-} \bar{b}+$ jets $\rightarrow 2 l^{-}+l^{+}+\bar{b}+\mathbb{E}_{T}+$ jets,

where the light jet can be faked as $b$-jet and the leptons may escape the detector.

In our simulation, we apply the following basic cuts on the signal and background events firstly

$$
\begin{gathered}
p_{T}^{l}>10 \mathrm{GeV}, \quad\left|\eta^{l}\right|<2.5, \quad p_{T}^{j}>20 \mathrm{GeV}, \\
\left|\eta^{j}\right|<5, \quad \Delta R(x, y)>0.4, \quad x, y=l, j .
\end{gathered}
$$

In order to get some hints of further cuts for reducing the backgrounds, we analysis the normalized distributions of $p_{T}^{l}$, $p_{T}^{j}, M_{j_{1}, j_{2}}$ and $E_{T}$ for the signals and backgrounds as shown in Fig. 7. Then, to get high statistical significance, a set of further cuts are given as followings.

- Cut 1: The normalized distributions of transverse momenta of leptons and jets for signals and backgrounds are shown in Fig. 7a, b, we can see that the transverse momenta of signal events are distributed mostly at large $p_{T}^{l, j}$ values, which are different from the distributions of background events. Then we require $p_{T}^{l}>70 \mathrm{GeV}$ and $p_{T}^{j}>70$ $\mathrm{GeV}$ to enhance the signal significance.

- Cut 2: In Fig. 7c, the background events have sharp peaks in the distributions of the invariant mass of lepton pairs. So the cut $M_{j_{1}, j_{2}}>100 \mathrm{GeV}$ is applied.

- Cut 3: Fig. 7d show the normalized distributions of $E_{T}$ which denotes the total transverse energy of leptons and 


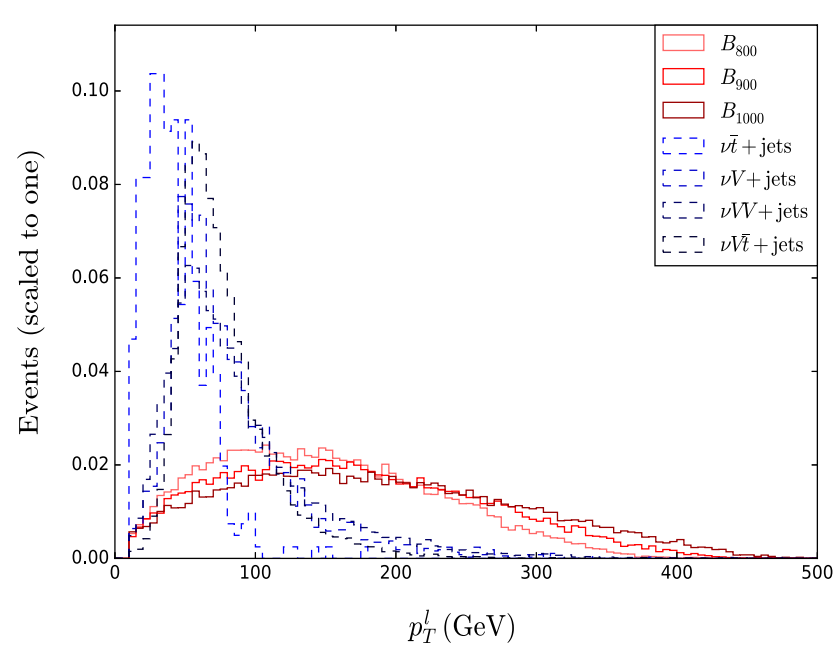

(a)

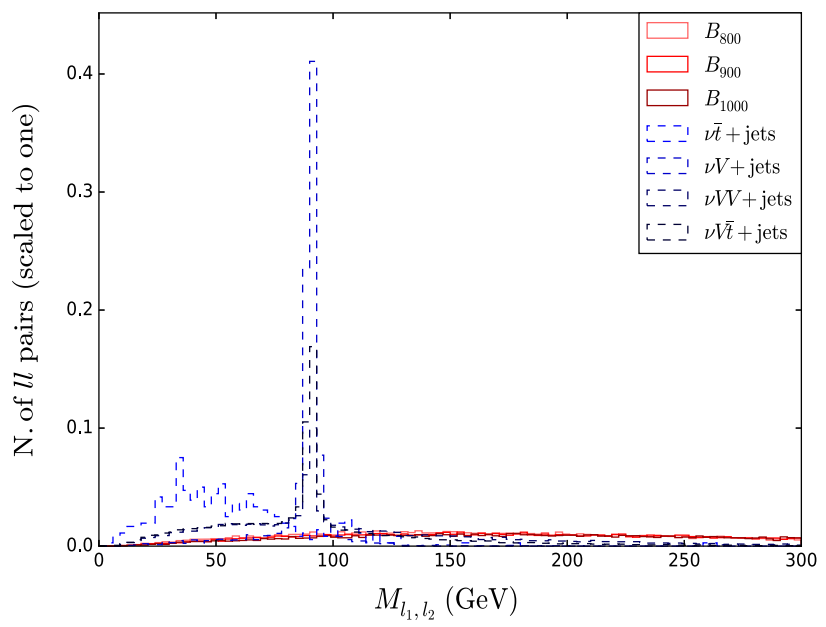

(c)

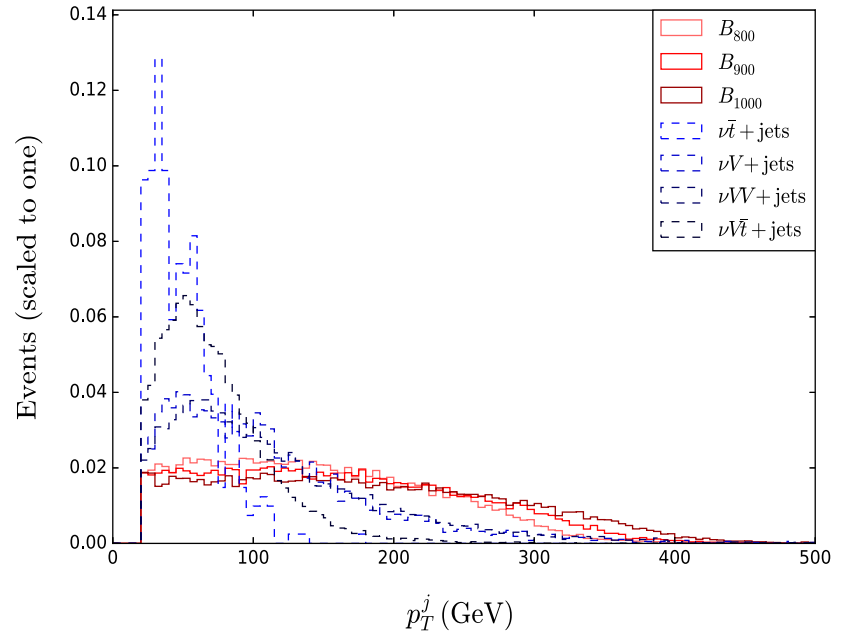

(b)

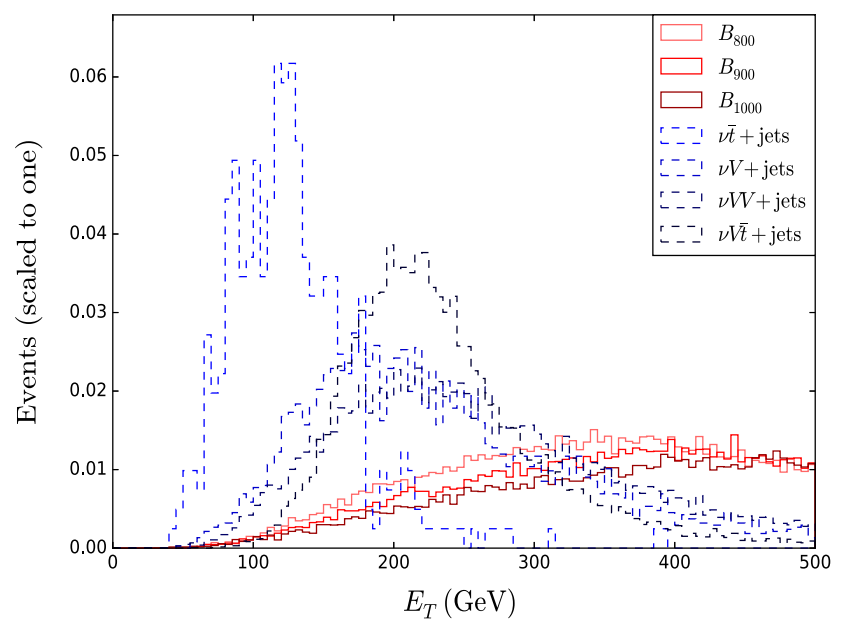

(d)

Fig. 7 Normalized distributions of $p_{T}^{l}, p_{T}^{j}, M_{j_{1}, j_{2}}$ and $E_{T}$ for signals and backgrounds at the LHeC with the integrated luminosity of $1000 \mathrm{fb}^{-1}$

Table 2 Same as Table 1, but for the fully leptonic channel

\begin{tabular}{|c|c|c|c|c|c|c|c|c|}
\hline & \multicolumn{3}{|c|}{ Signals } & \multicolumn{5}{|c|}{ Backgrounds } \\
\hline & $B_{800}$ & $B_{900}$ & $B_{1000}$ & $v \bar{t}+$ jets & $v V+$ jets & $v V V+$ jets & $v V \bar{t}+\mathrm{jets}$ & Total \\
\hline Basic cuts & 171.5 & 99.0 & 53.2 & 23230 & 116730 & 1374.8 & 108.9 & 141444 \\
\hline Cut 1 & 112.9 & 69.7 & 38.7 & 229.4 & 42667 & 521.1 & 25.6 & 43443 \\
\hline Cut 2 & 92.7 & 58.6 & 33.1 & 114.7 & 2391.1 & 347.7 & 14.8 & 2868.3 \\
\hline Cut 3 & 81.3 & 52.9 & 30.7 & 57.4 & 1304.2 & 244.9 & 8.1 & 1614.6 \\
\hline
\end{tabular}

jets. From these distributions, we can efficiently reduce the backgrounds by the cut: $E_{T}>300 \mathrm{GeV}$.

We summarize the numbers of the signal and background events at the $\operatorname{LHeC}(\sqrt{s}=1.98 \mathrm{TeV})$ with the integrated luminosity $\mathcal{L}=1000 \mathrm{fb}^{-1}$ in Table 2 . The values of $\mathrm{SS}$ can respectively reach about $2.0,1.3$ and 0.8 at the $\mathcal{L}=1000$ $\mathrm{fb}^{-1}$ for $M_{B}=800,900$ and $1000 \mathrm{GeV}$. And the $3 \sigma$ (left) and $5 \sigma$ (right) contour plots in the $R_{L}-\kappa_{B}$ plane are shown in Fig. 8. We can see that, for $R_{L}=0.5$ and $M_{B}=800$, 900 and $1000 \mathrm{GeV}, \mathrm{SS}$ can reach $3 \sigma$ when the values of $\kappa_{B}$ is about $0.107,0.132$ and 0.173 . Compared to the fully hadronic channel, the fully leptonic channel is not a good process to test the singlet VLQ- $B$ at the LHeC. 


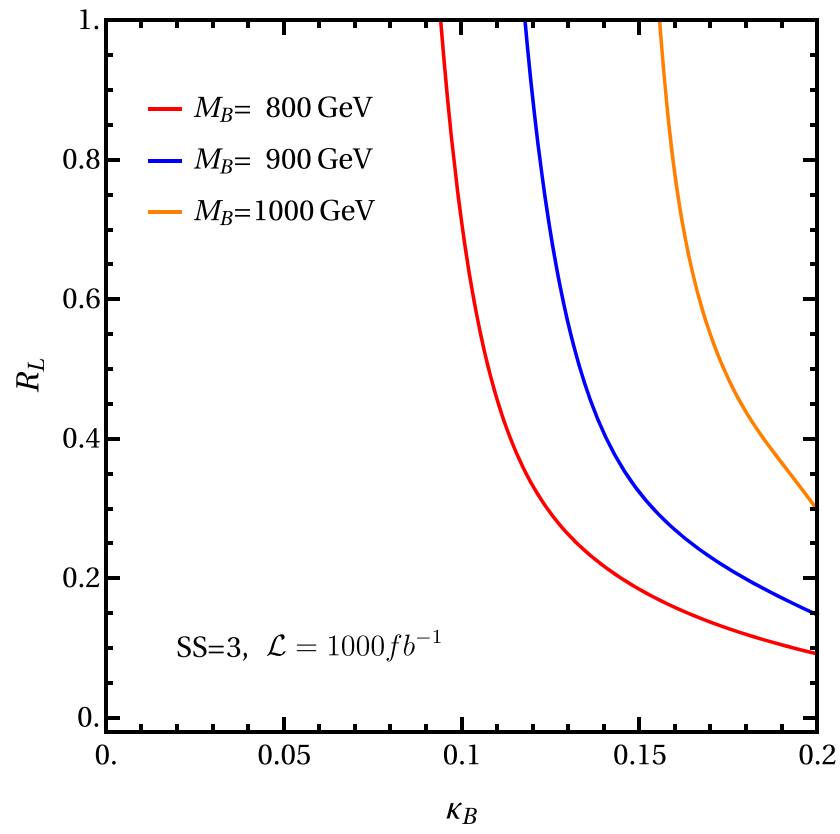

(a)

Fig. 8 Same as Fig. 5, but for the fully leptonic channel

\subsection{The semileptonic channel}

Now, we investigate the observability of the singlet VLQ- $B$ at the $\mathrm{LHeC}$ through the semileptonic decay channel

$$
\begin{aligned}
e^{-} p & \rightarrow v B\left(\rightarrow W^{-} t\right) \rightarrow v W^{-}\left(\rightarrow l_{i}^{-} \bar{v}_{i}\right) t\left(\rightarrow W^{+} b \rightarrow j j b\right) \\
& \rightarrow l^{-}+2 j+b+\mathbb{E}_{T} .
\end{aligned}
$$

For this channel, the typical signal is exactly one charged lepton, one $b$-jet, two jets (which coming from the top quark decay) and missing energy.

The dominant SM backgrounds come from the following processes:

- BKG1: $v \bar{t}+$ jets: $e^{-} p \rightarrow v \bar{t}+$ jets $\rightarrow v W^{-} \bar{b}+$ jets $\rightarrow$ $l^{-}+\bar{b}+\mathbb{E}_{T}+$ jets,

- BKG2: $v V+$ jets: $e^{-} p \rightarrow v V+$ jets $\rightarrow\left(l^{-}\right.$or $\left.l^{-} l^{+}\right)+$ $\mathbb{E}_{T}+$ jets,

- BKG3: $v V V+$ jets: $e^{-} p \rightarrow \nu V V+$ jets $\rightarrow\left(l^{-}\right.$or $\left.l^{-} l^{+}\right)+$ $\mathbb{E}_{T}+$ jets,

- BKG4: $v V \bar{t}+$ jets: $e^{-} p \rightarrow v Z \bar{t}+$ jets $\rightarrow v Z W^{-} \bar{b}+$ jets $\rightarrow 2 l^{-}+l^{+}+\bar{b}+\mathbb{E}_{T}+$ jets,

where one light jet might be faked as $b$-jet and the leptons may escape the detector.

We apply the following basic cuts on the signal and background events in our simulation:

$$
\begin{aligned}
& p_{T}^{l}>10 \mathrm{GeV}, \quad\left|\eta^{l}\right|<2.5, \quad p_{T}^{j}>20 \mathrm{GeV}, \quad\left|\eta^{j}\right|<5, \\
& \Delta R(x, y)>0.4, \quad x, y=l, j
\end{aligned}
$$

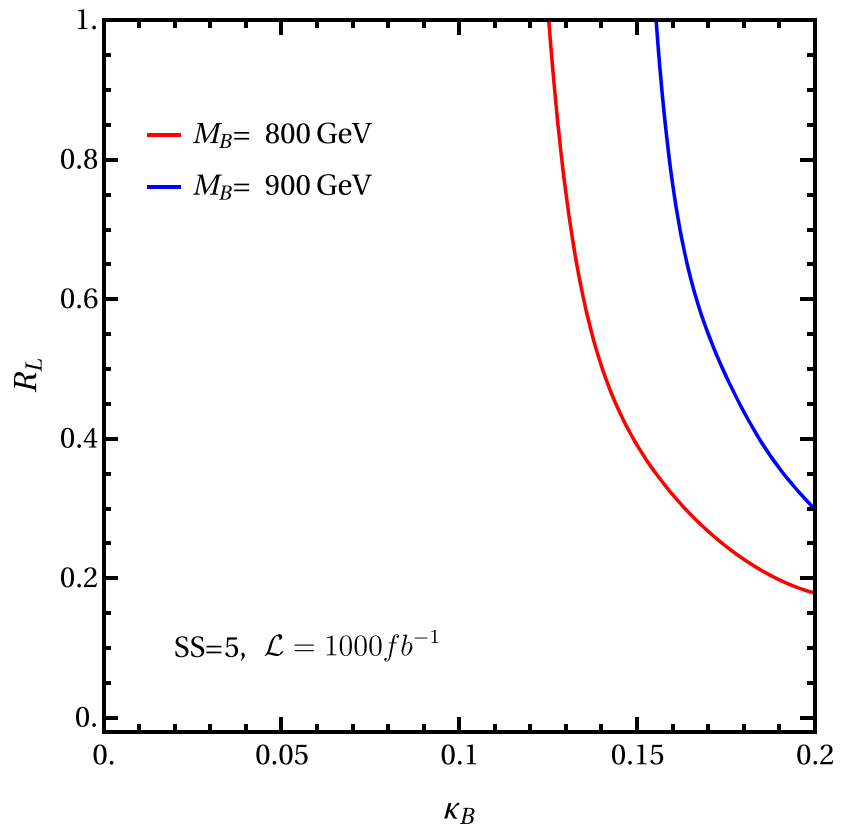

(b)
Further, we apply some general preselections as following.

To carry out the cut-based analysis, we discuss the normalized distributions of $\Delta R_{j_{1}, j_{2}}, p_{T}^{l}, M_{j_{1}, j_{2}}$ and $H_{T}$ for signals and backgrounds at the $\mathrm{LHeC}$ with the integrated luminosity of $1000 \mathrm{fb}^{-1}$ shown in Fig. 9. All cuts are applied one after the other in the order given in the following list.

- Cut 1 : In Fig. 9a, we show the normalized distributions of the particle separation of jets $\Delta R_{j_{1}, j_{2}}$ for signals and backgrounds. Since most jets of signals come from boosted object, they have much narrower separation. Based on the normalized distributions, we impose the first cut to get a high significance: $\Delta R_{j_{1}, j_{2}}<1.5$.

- Cut 2: Figure $9 \mathrm{~b}$ is the normalized distributions of the transverse momenta of leptons. Since the leptons of backgrounds most come from the decay of static $W$ or $Z$ bosons, the peaks local in half the mass of the $W$ or $Z$ boson. Thus cut $p_{T}^{l}>80 \mathrm{GeV}$ can reduce the backgrounds effectively.

- Cut 3: In Fig. 9c, the normalized distribution of $M_{j_{1}, j_{2}}$ is given. The peaks of signals are larger than those of backgrounds. Then the cut with $M_{j_{1}, j_{2}}>100 \mathrm{GeV}$ is selected.

- Cut 4: The Fig. 9d show the total hadronic energy $H_{T}$. Since the massive VLQ- $B$, the signal events should have higher hadronic energy than those of background events. Finally, we keep the events with $H_{T}>300 \mathrm{GeV}$. 


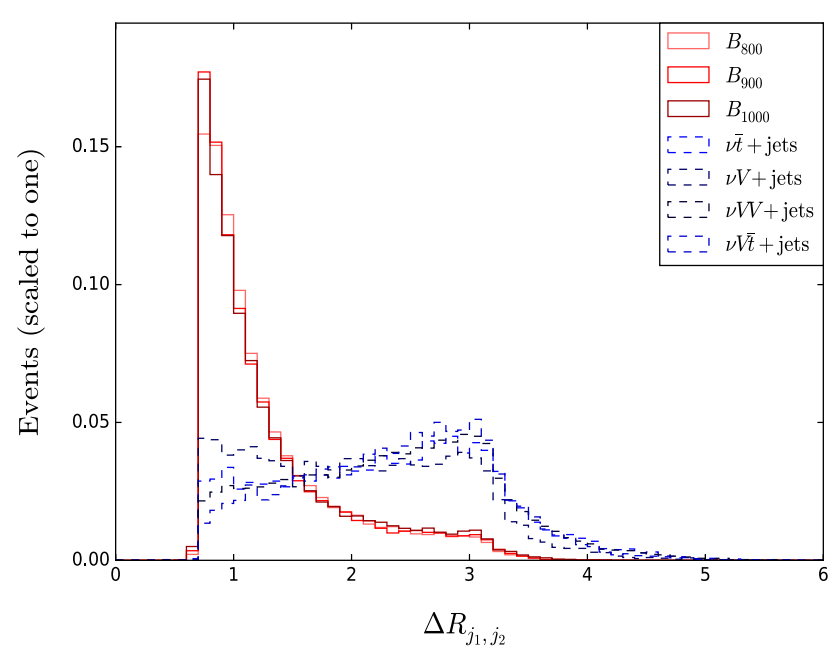

(a)

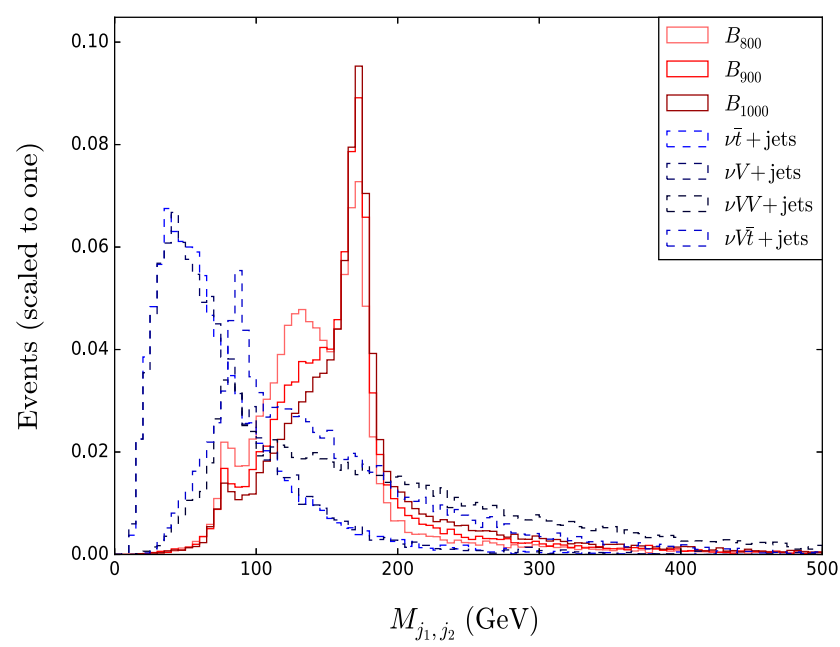

(c)

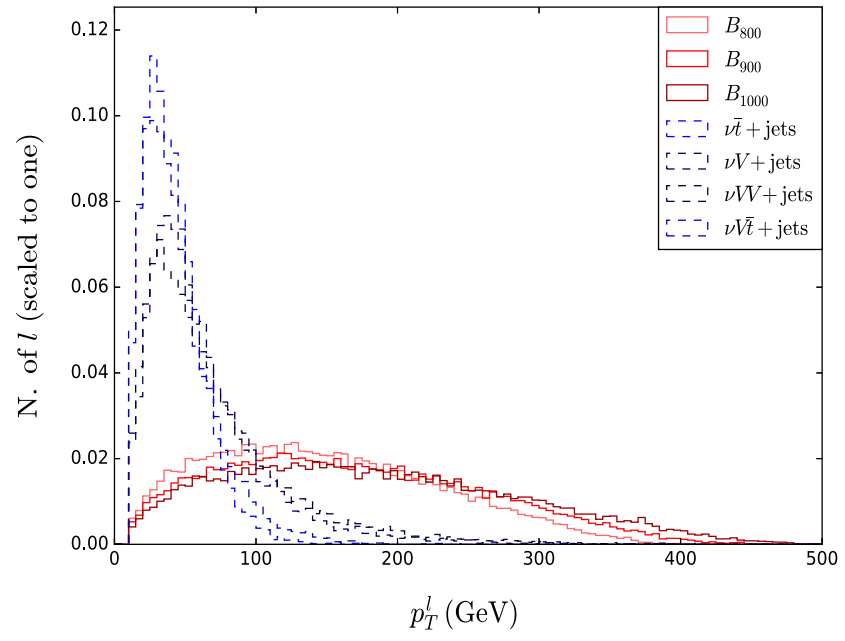

(b)

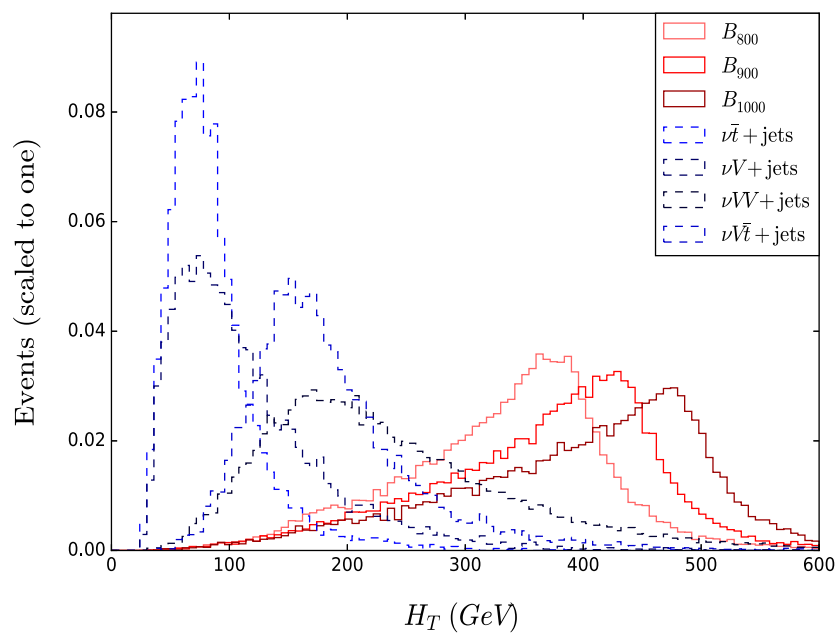

(d)

Fig. 9 Normalized distributions of $\Delta R_{j_{1}, j_{2}}, p_{T}^{l}, M_{j_{1}, j_{2}}$ and $H_{T}$ for signals and backgrounds at the LHeC with the integrated luminosity of 1000 $\mathrm{fb}^{-1}$

In Table 3, we show the numbers of the signal and background events at the $\mathrm{LHeC}(\sqrt{s}=1.98 \mathrm{TeV})$ with the integrated luminosity $\mathcal{L}=1000 \mathrm{fb}^{-1}$. After imposing the above selection cuts, the backgrounds are suppressed efficiently. The values of SS can respectively reach about 10.4, 7.5 and 4.6 at the $\mathcal{L}=1000 \mathrm{fb}^{-1}$ for $M_{B}=800,900$ and $1000 \mathrm{GeV}$.

Figure 10 shows the $3 \sigma$ (left) and $5 \sigma$ (right) contour plots in the $R_{L}-\kappa_{B}$ plane with three typical VLQ- $B$ mass at the LHeC with $\mathcal{L}=1000 \mathrm{fb}^{-1}$. For $R_{L}=0.5$ and $M_{B}=800$, 900 and $1000 \mathrm{GeV}$, SS reach $3 \sigma(5 \sigma)$ while the values of $\kappa_{B}$ achieve about $0.043(0.058), 0.053(0.070)$ and $0.071(0.093)$ separately.

From above discussions, we can see that, for single production of VLQ- $B$ at the $\mathrm{LHeC}$, it is possible to detect its signal via the fully hadronic, the fully leptonic and the semileptonic final states. However, the VLQ- $B$ which is the $S U(2)$ singlet with electric charge $-1 / 3$, can be more easy detected via the semileptonic decay channel at the LHeC.

\section{Conclusions}

In this paper, we study the discovery potential of the single production of VLQ- $B$ at the $\mathrm{LHeC}$, through three types of the characteristic signals, which come from the fully hadronic, the fully leptonic and the semileptonic decay channels. We focus our attention on the $S U(2)$ singlet VLQ- $B$ with electric charge $-1 / 3$ in a model-independent fashion. We investigate the observability of the VLQ- $B$ signal through these three decay channels at the $\mathrm{LHeC}$ with the integrated luminosity $\mathcal{L}=1000 \mathrm{fb}^{-1}$. In our numerical calculation, we obtain the $3 \sigma$ possible evidence region as well as the $5 \sigma$ discovery region, 
Table 3 Same as Table 1 but for the semileptonic channel

\begin{tabular}{llllllllll}
\hline & Signals & \multicolumn{7}{l}{ Backgrounds } \\
\cline { 2 - 3 } & $B_{800}$ & $B_{900}$ & $B_{1000}$ & & $v \bar{t}+$ jets & $\nu V+$ jets & $v V V+$ jets & $v V \bar{t}+$ jets & Total \\
\hline Basic cuts & 629.1 & 369.6 & 199.0 & 497564 & 159070 & 9306.5 & 828.8 & 666770 \\
Cut 1 & 472.9 & 278.2 & 147.2 & 114242 & 50103 & 1950.7 & 144.6 & 166441 \\
Cut 2 & 378.7 & 232.3 & 125.5 & 7539.8 & 13289 & 706.4 & 20.0 & 21555 \\
Cut 3 & 332.5 & 211.5 & 116.9 & 241.3 & 667.5 & 308.2 & 3.1 & 1220.1 \\
Cut 4 & 276.2 & 187.6 & 106.4 & 0 & 283.2 & 148.5 & 0.8 & 432.5 \\
\hline
\end{tabular}

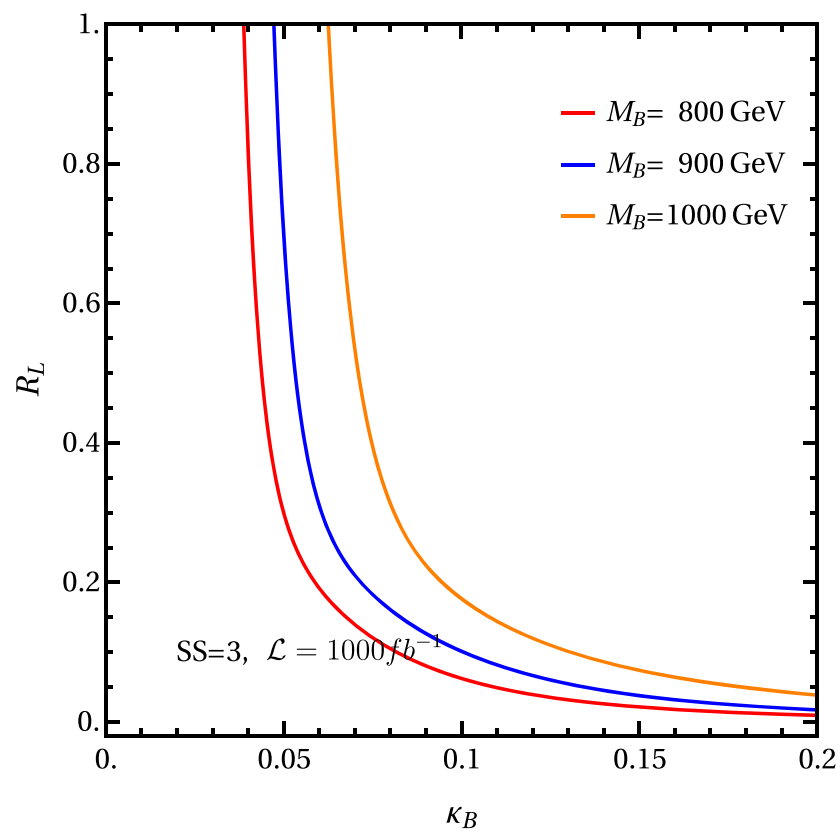

(a)

Fig. 10 Same as Fig. 5, but for the semileptonic channel

which are respectively presented in terms of parameter space regions for three typical masses $(800 \mathrm{GeV}, 900 \mathrm{GeV}, 1000$ $\mathrm{GeV}$ ). For $R_{L}=0.5$ and $M_{B}=800,900$ and $1000 \mathrm{GeV}$, the values of SS reach $3 \sigma$ when $\kappa_{B}$ is about $0.076,0.110$ and 0.156 in the fully hadronic channel, $0.107,0.132$ and 0.173 in the fully leptonic channel, and $0.043,0.053$ and 0.071 in the semileptonic channel. Thus, the possible signatures of the $S U(2)$ singlet VLQ- $B$ with electric charge $-1 / 3$ is easier detected via the process $e^{-} p \rightarrow v B\left(\rightarrow W^{-} t\right)$ in the semileptonic channel than other decay channels at the $\mathrm{LHeC}$. We expect our analysis can provide a complementary candidate to pursue searching for the singlet VLQ- $B$ at the $\mathrm{LHeC}$.

Acknowledgements This work was partially supported by the National Natural Science Foundation of China under Grants No. 11875157 and Grant No. 11847303.

Data Availability Statement This manuscript has no associated data or the data will not be deposited. [Authors' comment: The curve graphs in the paper present all our data.]

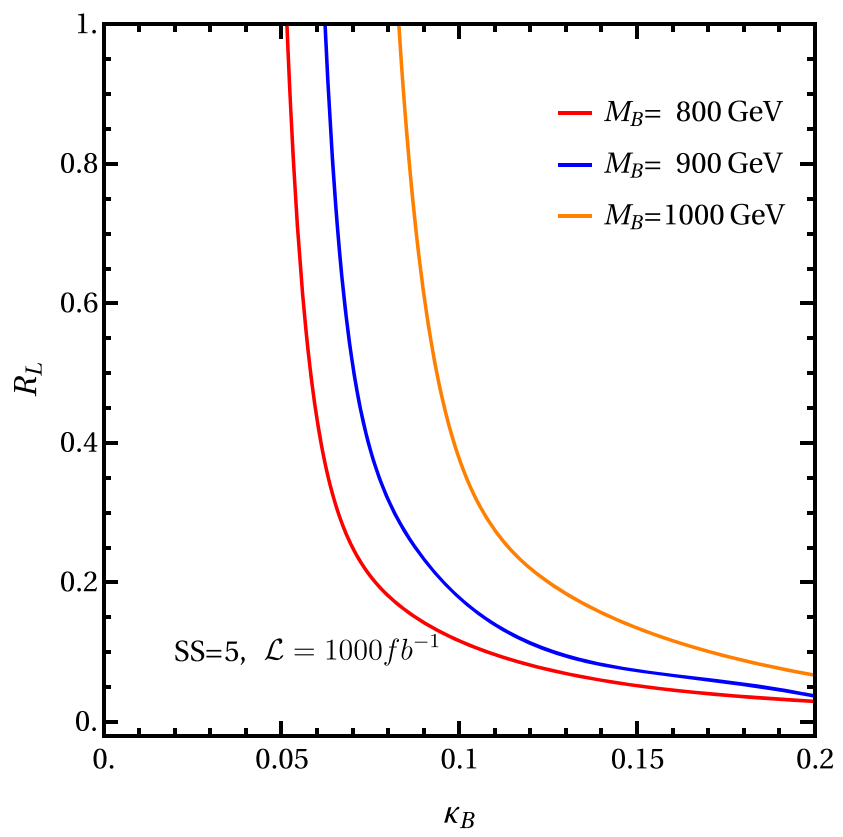

(b)

Open Access This article is licensed under a Creative Commons Attribution 4.0 International License, which permits use, sharing, adaptation, distribution and reproduction in any medium or format, as long as you give appropriate credit to the original author(s) and the source, provide a link to the Creative Commons licence, and indicate if changes were made. The images or other third party material in this article are included in the article's Creative Commons licence, unless indicated otherwise in a credit line to the material. If material is not included in the article's Creative Commons licence and your intended use is not permitted by statutory regulation or exceeds the permitted use, you will need to obtain permission directly from the copyright holder. To view a copy of this licence, visit http://creativecomm ons.org/licenses/by/4.0/.

Funded by $\mathrm{SCOAP}^{3}$.

\section{References}

1. G. Aad et al. [ATLAS Collaboration], Phys. Lett. B 716, 1 (2012)

2. S. Chatrchyan et al. [CMS Collaboration], Phys. Lett. B 716, 30 (2012)

3. A. De Simone, O. Matsedonskyi, R. Rattazzi, A. Wulzer, J. High Energy Phys. 1304, 004 (2013) 
4. J.A. Aguilar-Saavedra, R. Benbrik, S. Heinemeyer, M. PérezVictoria, Phys. Rev. D 88, 094010 (2013)

5. N. Arkani-Hamed, A.G. Cohen, E. Katz, A.E. Nelson, J. High Energy Phys. 0207, 034 (2002)

6. N. Arkani-Hamed, A.G. Cohen, E. Katz, A.E. Nelson, T. Gregoire, J.G. Wacker, J. High Energy Phys. 0208, 021 (2002)

7. M. Schmaltz, Nucl. Phys. Proc. Suppl. 117, 40 (2003)

8. M. Schmaltz, D. Tucker-Smith, Ann. Rev. Nucl. Part. Sci. 55, 229 (2005)

9. D.B. Kaplan, H. Georgi, S. Dimopoulos, Phys. Lett. B 136, 187 (1984)

10. K. Agashe, R. Contino, A. Pomarol, Nucl. Phys. B 719, 165 (2005)

11. C. Anastasiou, E. Furlan, J. Santiago, Phys. Rev. D 79, 075003 (2009)

12. M. Low, A. Tesi, L.T. Wang, Phys. Rev. D 91, 095012 (2015)

13. L. Randall, R. Sundrum, Phys. Rev. Lett. 83, 3370 (1999)

14. H.C. Cheng, B.A. Dobrescu, C.T. Hill, Nucl. Phys. B 589, 249 (2000)

15. F. del Aguila, M.J. Bowick, Nucl. Phys. B 224, 107 (1983)

16. J.A. Aguilar-Saavedra, J. High Energy Phys. 0911, 030 (2009)

17. F. del Aguila, J. Santiago, Phys. Lett. B 493, 175 (2000)

18. P.H. Frampton, P. Hung, M. Sher, Phys. Rep. 330, 263 (2000)

19. J.L. Hewett, T.G. Rizzo, Phys. Rep. 183, 193 (1989)

20. R. Contino, L. Da Rold, A. Pomarol, Phys. Rev. D 75, 055014 (2007)

21. M. Carena, E. Ponton, J. Santiago, C.E. Wagner, Nucl. Phys. B 759, 202 (2006)

22. J. Aguilar-Saavedra, J. Alonso-González, L. Merlo, J. No, Phys. Rev. D 101, 035015 (2020)

23. B. Fuks, H.S. Shao, Eur. Phys. J. C 77, 135 (2017)

24. J.M. Campbell, R. Frederix, F. Maltoni, F. Tramontano, J. High Energy Phys. 0910, 042 (2009)

25. Y. Okada, L. Panizzi, Adv. High Energy Phys. 2013, 364936 (2013)

26. M. Buchkremer, G. Cacciapaglia, A. Deandrea, L. Panizzi, Nucl. Phys. B 876, 376 (2013)

27. A. Anandakrishnan, J.H. Collins, M. Farina, E. Kuflik, M. Perelstein, Phys. Rev. D 93, 075009 (2016)

28. S. Yang, J. Jiang, Q.S. Yan, X. Zhao, J. High Energy Phys. 1409, 035 (2014)

29. J. Reuter, M. Tonini, J. High Energy Phys. 1501, 088 (2015)

30. H.J. He, C.T. Hill, T.M.P. Tait, Phys. Rev. D 65, 055006 (2002)

31. H.J. He, T.M.P. Tait, C.P. Yuan, Phys. Rev. D 62, 011702 (2000)

32. X.F. Wang, C. Du, H.J. He, Phys. Lett. B 723, 314 (2013)

33. D. Barducci et al., J. High Energy Phys. 1412, 080 (2014)

34. H.J. He, Z.Z. Xianyu, JCAP 1410, 019 (2014)

35. B. Yang, B. Hou, H. Zhang, N. Liu, Phys. Rev. D 99, 095002 (2019)

36. D. Liu, L.T. Wang, K.P. Xie, J. High Energy Phys. 1901, 157 (2019)

37. G. Cacciapaglia, A. Deandrea, N. Gaur, D. Harada, Y. Okada, L. Panizzi, J. High Energy Phys. 11, 055 (2018)

38. A. Buckley, J.M. Butterworth, L. Corpe, D. Huang, P. Sun, arXiv:2006.07172

39. N. Vignaroli, J. High Energy Phys. 1207, 158 (2012)

40. J. Nutter, R. Schwienhorst, D.G.E. Walker, J.H. Yu, Phys. Rev. D 86, 094006 (2012)

41. K. Cheung, W. Keung, C. Lu, P. Tseng, arXiv:2001.02853
42. X. Gong, C.X. Yue, Y.C. Guo, Phys. Lett. B 793, 175 (2019)

43. M. Aaboud et al. [ATLAS Collaboration], Phys. Rev. D 98, 092005 (2018)

44. M. Aaboud et al. [ATLAS Collaboration], Phys. Rev. Lett. 121, 211801 (2018)

45. A.M. Sirunyan et al. [CMS Collaboration], J. High Energy Phys. 1808, 177 (2018)

46. M. Aaboud et al. [ATLAS Collaboration], J. High Energy Phys. 1808, 048 (2018)

47. A.M. Sirunyan et al. [CMS Collaboration], J. High Energy Phys. 1806, 031 (2018)

48. J.L. Abelleira Fernandez et al. [LHeC Study Group], J. Phys. G 39, 075001 (2012)

49. Y.B. Liu, Nucl. Phys. B 923, 312 (2017)

50. Y.J. Zhang, L. Han, Y.B. Liu, Phys. Lett. B 768, 241 (2017)

51. L. Han, Y.J. Zhang, Y.B. Liu, Phys. Lett. B 771, 106 (2017)

52. Y. Okada, L. Panizzi, Adv. High Energy Phys. 2013, 364936 (2013)

53. M. Buchkremer, G. Cacciapaglia, A. Deandrea, L. Panizzi, Nucl. Phys. B 876, 376 (2013)

54. A. Atre, M. Carena, T. Han, J. Santiago, Phys. Rev. D 79, 054018 (2009)

55. B.W. Lee, C. Quigg, H.B. Thacker, Phys. Rev. D 16, 1519 (1977)

56. M. Tanabashi et al. [Particle Data Group], Phys. Rev. D 98, 030001 (2018)

57. http://feynrules.irmp.ucl.ac.be/wiki/VLQ_bsingletvl

58. N.D. Christensen, C. Duhr, Comput. Phys. Commun. 180, 1614 (2009)

59. N.D. Christensen, P. de Aquino, C. Degrande, C. Duhr, B. Fuks, M. Herquet, F. Maltoni, S. Schumann, Eur. Phys. J. C 71, 1541 (2011)

60. A. Alloul, N.D. Christensen, C. Degrande, C. Duhr, B. Fuks, Comput. Phys. Commun. 185, 2250 (2014)

61. J. Alwall, M. Herquet, F. Maltoni, O. Mattelaer, T. Stelzer, J. High Energy Phys. 1106, 128 (2011)

62. J. Alwall et al., J. High Energy Phys. 1407, 079 (2014)

63. J. Pumplin, A. Belyaev, J. Huston, D. Stump, W.K. Tung, J. High Energy Phys. 0602, 032 (2006)

64. T. Sjostrand, S. Mrenna, P.Z. Skands, J. High Energy Phys. 0605, 026 (2006)

65. M. Klein, LHeC Detector Design, 25th International Workshop on Deep Inelastic Scattering, Birmingham (2017). https://indico.cern. ch/event/568360/contributions/2523637/

66. M. Cacciari, G.P. Salam, G. Soyez, J. High Energy Phys. 0804, 063 (2008)

67. E. Conte, B. Fuks, G. Serret, Comput. Phys. Commun. 184, 222 (2013)

68. E. Conte, B. Dumont, B. Fuks, C. Wymant, Eur. Phys. J. C 74, 3103 (2014)

69. B. Dumont et al., Eur. Phys. J. C 75, 56 (2015)

70. S. Chatrchyan et al. [CMS Collaboration], arXiv:1211.4462

71. S. Hoeche, F. Krauss, N. Lavesson, L. Lonnblad, M. Mangano, A. Schalicke, S. Schumann, arXiv:hep-ph/0602031 